\title{
Case report on a patient with lupus panniculitis
}

\author{
Agata Bednarek, Leszek Bartoszak, Włodzimierz Samborski \\ Department of Rheumatology and Rehabilitation, Poznan University of Medical Sciences, Poznan, Poland \\ Head of Department: Prof. Włodzimierz Samborski MD, PhD
}

Postep Derm Alergol 2015; XXXII, 1: 59-62

DOI: $10.5114 /$ pdia.2014.40958

\begin{abstract}
Lupus panniculitis is a rare variant of lupus erythematosus. It may occur as a separate disease or coexist with systemic or discoid lupus erythematosus. It is characterized by persistent, tender and hard nodules localised on the face, arms, shoulders, breast and buttocks. Healing of lesions is associated with scarring, lipoatrophy and rarely ulceration. Treatment of lupus panniculitis depends on disease advancement or concomitance of additional lupus erythematosus symptoms. We report a case of a 44-year-old patient with lupus panniculitis treated with chloroquine and glucocorticosteroids, including high dose infusions. Despite intense treatment, the patient developed symptoms that suggested a diagnosis of systemic lupus erythematosus.
\end{abstract}

Key words: lupus panniculitis, systemic lupus erythematosus, diagnosis, treatment.

\section{Case report}

In April 2008, a 44-year-old woman was admitted to the Rheumatology and Rehabilitation University Hospital with the aim of establishing a diagnosis of suspected systemic lupus erythematosus (SLE). The main complaint of the patient on admission to hospital was the occurrence of painful, bluish-red skin lesions causing irregular concavities and palpable subcutaneous nodules. The patient developed the first lesion of this type in January 2007. It was painful and located on the lateral surface of the left arm. As a result, the patient was hospitalised in the Dermatological Ward. As far as deviations from the norm are concerned, tests conducted during the hospitalisation revealed elevated erythrocyte sedimentation rate (ESR) $-18 \mathrm{~mm} / \mathrm{h}$ and leucopenia $-3.7 \times 10^{9} / \mathrm{l}$. Antibiotic treatment was applied but no improvement occurred and the patient was discharged from hospital with the suspicion of erythema nodosum. In March 2007, the evaluation of the dermal specimen showed thinning of epidermis and inflammatory infiltrations around minor blood vessels in the hypodermis. The histopathological examination corresponded with the diagnosis of erythema nodosum. Another hospitalisation took place in June 2007 in the Dermatological University Hospital. On admission, erythematous-infiltrated lesions were located on the lateral surfaces of arms, thighs and on the anterior surfaces of the knee joints. Additional tests and examinations were conducted and again revealed leucopenia $3.5 \times 10^{9} / \mathrm{l}$. The ESR value was $40 \mathrm{~mm} / \mathrm{h}$. Antinuclear antibodies (ANA) were positive with the titre above 1 : 640 and centromere pattern. In histopathological examination, the picture was consistent with lupus panniculitis, whereas in the immunopathological examination of non-lesional skin specimens (lupus band test), the presence of igm deposits along the dermoepidermal junction was confirmed without the presence of IgG, IgA and C3 deposits. The final diagnosis was lupus panniculitis and further treatment in the Dermatological Out-Patient Clinic was planned. In order to determine whether there are any contraindications to treatment with chloroquine, a visit to the Ophthalmological Out-Patient Clinic was also recommended. After the consultation $250 \mathrm{mg}$ chloroquine a day was administered. The patient was told to avoid sun exposure and use high factor sunscreens. In July 2007, the deterioration of general feeling occurred - temperature of $39^{\circ} \mathrm{C}$ for 2 days and a burning sensation in the throat and oesophagus persisted. Methylprednisolone in dose $4 \mathrm{mg}$ a day orally was added to previous chloroquine treatment. The fever was reduced and the patient showed slight improvement.

Address for correspondence: Leszek Bartoszak MD, Department of Rheumatology and Rehabilitation, Poznan University of Medical Sciences, 49 Przybyszewskiego St, 60-856 Poznan, Poland, phone: +48 603740 510, phone/fax: 48618310244 ,

e-mail: leszek.bartoszak@gmail.com

Received: 29.09.2013, accepted: 2.12.2013. 


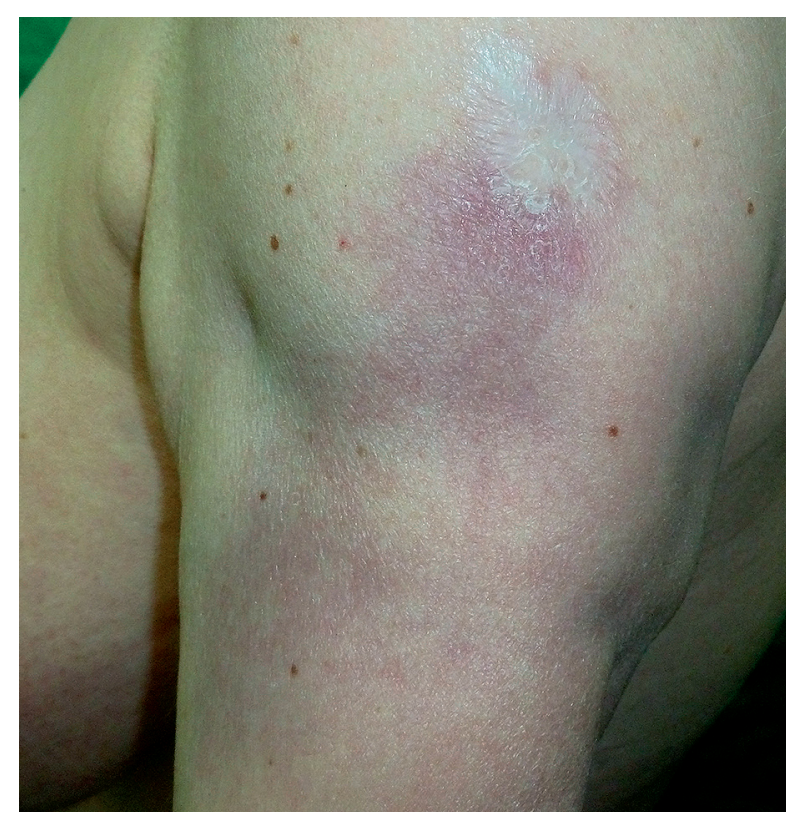

Figure 1. Bluish-red skin lesions, scarring and irregular concavities localised on the lateral surface of the left arm

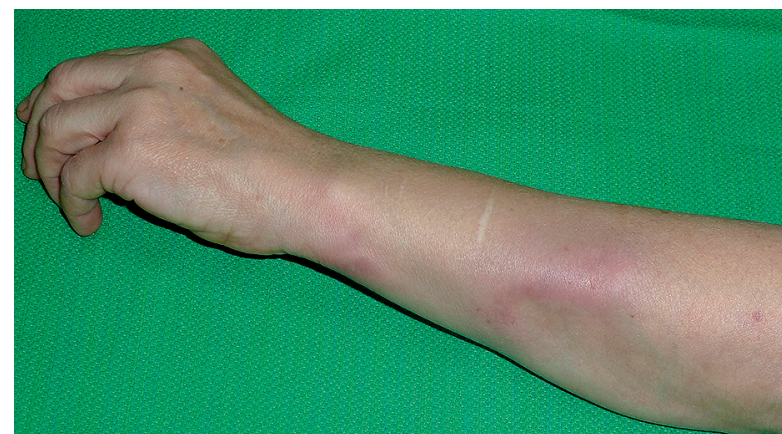

Figure 2. Bluish-red subcutaneous nodules and lipoatrophy localized on the left forearm

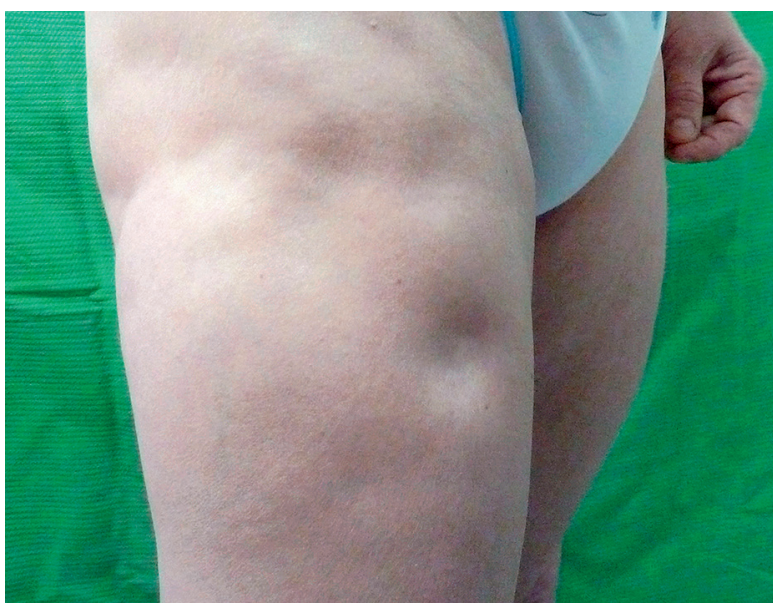

Figure 3. Disseminated concavities localised on the lateral surface of the right thigh
Nine months later, on admission to the Rheumatology and Rehabilitation University Hospital, the patient complained of the sacral spine and right arm pain, as well as of general symptoms such as general weakness and weight loss of $10 \mathrm{~kg}$ throughout the last 6 months. On examination, subcutaneous nodules of 2 to $4 \mathrm{~cm}$ in diameter, located in irregular concavities within the lateral surfaces of both arms, frontal surfaces of forearms and the lateral surfaces of thighs were observed (Figures 1-3). The lesions were not painful. The examination revealed also oedema and tenderness in metacarpophalangeal joints bilaterally. The indirect immunofluorescence test revealed positive ANA with the titre of $1: 640$. Euroline ANA profile was positive for anti-Ro-52 and anti-centromere antibodies. Other additional tests showed ESR - $24 \mathrm{~mm} / \mathrm{h}$, free serum thyroxin - decreased (1.69 pmol/l), thyroid-stimulating hormone - in norm and positive tuberculin sensitivity test. Chest $X$-ray showed no features of the tuberculosis process. During dermatological consultation, the clinical picture was confirmed to be consistent with lupus panniculitis. For 3 consecutive days 500 mg infusions of methylprednisolone, and then oral methylprednisolone treatment $4 \mathrm{mg}$ a day, was performed. The patient was discharged and recommended to continue the treatment in the Clinic in 8 weeks' time. After 2 months, on admission, physical condition of the patient was good. However, lesions were still present and the patient complained of painful knee joints and mood deterioration caused by the disease. For the latter reason, the patient was consulted in the Psychiatry Clinic, where clomipramine was prescribed, which she did not take. Additional tests revealed ESR normalisation. Three infusions of methylprednisolone were repeated (total $1500 \mathrm{mg}$ ) and then oral methylprednisolone therapy was continued for 2 months. After that, the patient was admitted to hospital. On discharge she did not complain of arthralgia any longer, lesions were paler and no new lesions were observed. During the next hospitalisation in the University Hospital in July 2010, the patient complained of general feeling deterioration, recurrent weakness correlated with the widespread pain and arthralgia, frequent headaches, hyperhidrosis and occasional nausea. New nodular lesions localised in previously occupied areas were hard, painful, bluish, from 0.5 to $3 \mathrm{~cm}$ in diameter and showed tendency to group and coalesce. Additionally, the skin of the limbs was covered with numerous extravasations, which were soft and painful. Tests revealed slightly elevated ESR up to $22 \mathrm{~mm} / \mathrm{h}$, positive ANA indirect immunofluorescence test with the titre of $1: 160$ and speckled pattern, presence of anti-dsdna (+), anti-RNP (++), anti-Ro-52 (+++), anti-centromere $(++)$ and anti-Sm (trace reaction) antibodies in the ANA profile. At this time the patient fulfilled the following ACR diagnostic criteria for SLE - arthritis, leucopenia, the presence of ANA in indirect immunofluorescence test and the presence of anti-dsdna and anti-Sm antibodies in the ANA 
profile. Once again infusions of methylprednisolone were administered (500-500-250 mg) for 3 consecutive days. The patient was discharged in good general condition with a diagnosis of SLE and was recommended to come to the University Hospital again after 3 months for further treatment.

\section{Discussion}

Kaposi was most probably the first author who described the disease. In 1883, he characterised subcutaneous lesions co-occurring in lupus erythematosus. Lupus panniculitis, also called lupus profundus, is a relatively rare abnormality of connective tissue. It may occur as a separate disease or coexist with SLE or discoid lupus erythematosus (DLE) [1]. Lupus panniculitis occurs twice more frequently as a separate disease than in association with SLE or DLE [2]. On the other hand, 1-3\% of patients suffering from SLE and up to 10\% of those suffering from DLE develop lupus panniculitis [1, 3]. It may lead to the atrophy of the skin and hypodermis and sometimes to ulceration [1]. The course of the disease is relapsing and new lesions may reoccur in the previously afflicted areas or appear in new regions. Lesions are most frequently localised in the region of the arms, shoulders, face, scalp and buttocks, which may make diagnosis easier. New lesions are tender, reddish or purple, hard papules which may look like DLE lesions. Healing of lesions is associated with scarring and lipodystrophy, which leads to concavities in the skin that become a cosmetic problem to patients. This may bring mood deterioration or even depression and may require psychiatric treatment [4].

To establish diagnosis, the histopathological examination of lesional skin specimen is the golden standard. Two subtypes of panniculitis, namely septal and lobular, are distinguished. In the former, the intralobular septa of adipose tissue are affected by necrosis, whereas in the latter - the fat lobules. In practice, both patterns occur in the majority of patients simultaneously as the inflammatory infiltration is not strictly separated. In the dermal specimen of a lupus panniculitis patient, features of adipose tissue inflammation, such as lymphocyte, histiocyte and plasma cell infiltration or vascular wall thickening are visible [1, 5]. Intralobular septa may include hyalinised collagen. The older lesions may contain calcifications [6]. Lupus band test is yet another tool useful for diagnosing the disease. The test is carried out upon non-lesional skin biopsy and the positive result, which indicates the deposits of IgG, IgM and C3 antibodies, is obtained in 36-70\% of cases [7,8]. Unfortunately, the biopsy does not give information on how much the hypodermis is affected. In the future, it is likely to use computer tomography as a method revealing lesions and their scope [9]. The lesions are presented as high-density changes. As far as differentiating is concerned, various forms of panniculitis associated with vascular inflammation, diabetic microan- giopathy, venous insufficiency, infectious lesions, some forms of lymphoma (especially subcutaneous T-cell lymphoma) or post-traumatic adipose tissue necrosis should be taken into account. It may rarely be a manifestation of different rheumatic disorders, e.g. scleroderma or polymyositis [10-12].

The treatment of lupus profundus is at times difficult. There are no specific indications and medication schemes in lupus panniculitis treatment. Local treatment consists in using topical glucocorticosteroids and lubricant ointments. Also injections of glucocorticosteroids into lesional areas were considered as a therapeutic method [13]. Antimalarial drugs are believed to bring improvement in mild cases of separate lupus panniculitis. Chloroquine in dose 250-500 mg a day and hydroxychloroquine (not registered in Poland) in dose 200-400 mg a day is usually recommended. In cases with co-occurring SLE, antimalarial medications and glucocorticosteroids are most commonly used. Any attempt to discontinue immunotherapy altogether is always associated with the risk of lesion recurrence. In recurrence or more aggressive lupus panniculitis cases also methotrexate, cyclophosphamide and cyclosporine A are used [4, 14, 15]. Thalidomide may be used as alternative treatment in patients who do not respond to other forms of therapy. However, its application necessitates that the patient be strictly monitored with respect to possible neuropathies, which occur in $70 \%$ of cases and are considered to be irreversible $[16,17]$. Cases of lupus panniculitis improvement after disulone therapy were documented [18].

\section{Conclusions}

In the reported case, treatment strategy was different, namely methylprednisolone was administered. When lesions were aggravated and the patient's general feeling deteriorated, infusions of methylprednisolone were administered for 3 consecutive days in doses varying from 250 to $500 \mathrm{mg} / \mathrm{administration.} \mathrm{As} \mathrm{far} \mathrm{as} \mathrm{oral}$ glucocorticosteroids are concerned, doses were relatively low, but allowed the patient to control disease symptoms quite well. Nevertheless, in spite of the immunosuppressive treatment, the ANA profile conducted 1 year after treatment confirmed the presence of SLE specific anti-dsDNA antibodies, which together with the previous record of leucopenia and another symptoms, suggests the diagnosis of systemic lupus erythematosus associated with lupus panniculitis.

\section{Conflict of interest}

The authors declare no conflict of interest.

\section{References}

1. Martens PB, Moder KG, Ahmed I. Lupus panniculitis: clinical perspectives from a case series. J Rheumatol 1999; 26: 68-72. 
2. Callen JP. Cutaneous lupus erythematosus: a personal approach to management. Australas J Dermatol 2006; 47: 13-27.

3. Requena L, Sanches Yus E. Panniculitis. Part II. Mostly lobular panniculitis. J Am Acad Dermatol 2001; 45: 325-61.

4. Grossberg E, Scherschum L, Fivenson DP. Lupus profundus: not a benign disease. Lupus 2001; 10: 514-6.

5. Sontheimer RD, McCauliffe DP. Lupus-specific skin disease (cutaneous LE). In: Dubious'lupus erythematosus. Wallance DJ, Hahn BH (eds.). Lippincott Williams and Wilkins, Philadelphia 2007; 576-620.

6. Mckee PH. Inflammatory disease of the subcutaneous fat. In: Pathology of the skin with clinical correlations. Mckee PH, Calonje E, Granter SR (eds.). Elsevier, London 2005; 379-82.

7. Massone C, Kodama K, Salmhofer W, et al. Lupus erythematosus panniculitis (lupus profundus): clinical, histopathological, and molecular analysis of nine cases. J Cutan Pathol 2005; 32: 396-404.

8. Ng PP, Tan SH, Tan T. Lupus erythematosus panniculitis: a clinicopathologic study. Int I Dermatol 2002; 41: 488-90.

9. Kato T, Nakajima A, Kanno T, et al. Clinical utility of computed tomographic scanning for the evaluation of lupus profundus in two patients with systemic lupus erythematosus. Mod Rheumatol 2009; 19: 91-5.

10. Cassis TB, Fearneyhough PK, Callen JP. Subcutaneous panniculitis-like T cell lymphoma with vacuolar interface dermatitis resembling lupus erythematosus panniculitis. J Am Acad Dermatol 2004; 50: 465-9.

11. Almeida MS, Lima SC, Carvalho LL, et al. Panniculitis - an unusual cutaneous manifestation of systemic sclerosis. J Cutan Pathol 2010; 37: 1170-3.

12. Diaz-Cascajo C, Borghi S. Subcutaneous pseudomembranous fat necrosis: new observations. J Cutan Pathol 2002; 29: 5-10.

13. Yell JA, Burge SM. Lupus erythematosus profundus treated with clobetasol propionate under a hydrocolloid dressing. $\mathrm{Br}$ J Dermatol 1993; 128: 103.

14. Wazniacka A, Salamon M, Lesiak A, et al. The dynamism of cutaneous lupus erythematosis: mild discoid lupus erythematosus evolving into SLE with SCLE and treatment-resistant lupus panniculitis. Clin Rheumatol 2007; 26: 1176-9.

15. Saeki Y, Ohshima S, Kurimoto I, et al. Maintaining remission of lupus erythematosus profundus (LEP) with cyclosporine A. Lupus 2000; 9: 390-2.

16. Wu JJ, Huang DB, Pang KR, et al. Thalidomide: dermatological indications, mechanisms of action and side-effects. $\mathrm{Br}$ J Dermatol 2005; 153: 254-73.

17. Burrows NP, Walport MJ, Hammond AH, et al. Lupus erythematosus profundus with partial C4 deficiency responding to thalidomide. Br J Dermatol 1991; 125: 62-7.

18. Yamada Y, Dekio S, Jidoi J, Ozasa S. Lupus erythematosus profundus: report of a case treated with dapsone. J Dermatol 1989; 16: 379-82. 